Catherine E. Hassett, DO
Cerebrovascular Center, Neurological Institute,
Cleveland Clinic
Aron Gedansky, MD

Cerebrovascular Center, Neurological Institute,

Cleveland Clinic
Ibrahim Migdady, MD

Division of Neurocritical Care, Department of Neurology, Massachusetts General Hospital, Harvard Medical School Boston, MA
Adarsh Bhimraj, MD

Section Head-Neuroinfections, Department of Infectious Disease and Department of Neurology, Cleveland Clinic; Clinical Assistant Professor Cleveland Clinic Lerner College of Medicine of Case Western Reserve University, Cleveland, $\mathrm{OH}$
Ken Uchino, MD

Cerebrovascular Center, Neurological Institute,

Cleveland Clinic; Professor, Cleveland Clinic Lerner

College of Medicine of Case Western Reserve

University, Cleveland, $\mathrm{OH}$
Sung-Min Cho, DO

Departments of Neurology, Neurological Intensive

Care, Anesthesiology, and Critical Care Medicine,

Johns Hopkins University School of Medicine,

Baltimore, MD

\title{
Neurologic complications of COVID-19
}

\section{ABSTRACT}

Patients with COVID-19 have a fairly high risk of neurologic complications, including encephalopathy, stroke, central nervous system infection, seizures, and neuromuscular diseases. Many report losing their senses of smell and taste, and many survivors report lingering neurocognitive impairment. The diagnosis and treatment of these complications does not differ from that in other patients, although sophisticated testing may not be readily available for a patient in intensive care and respiratory isolation. Clinicians should therefore be alert to these complications.

\section{KEY POINTS}

Human coronaviruses, including SARS-CoV-2, can be neurotropic.

Commonly reported neurologic complications in patients infected with SARS-CoV-2 include encephalopathy, neuromuscular disorders, and acute cerebrovascular disorders.

Other complications such as postinfectious demyelination, encephalitis, and seizures are likely underreported given the inability to obtain further diagnostic information, such as cerebrospinal fluid sampling and electroencephalographic monitoring.

Long-term neurocognitive outcomes have yet to be established in COVID-19 survivors.

Clinicians should have a high clinical suspicion for associated neurologic complications in a COVID-19 patient.

The authors report no relevant financial relationships which, in the context of their contributions, could be perceived as a potential conflict of interest.

doi:10.3949/ccjm.87a.ccc058
T IKE OTHER MEMBERS of the coronavirus family, severe acute respiratory syndrome coronavirus 2 (SARS-CoV-2) can infect the nervous system. Coronaviruses share several features, including the large spike glycoproteins that inspired their name corona, Latin for crown. These spike glycoproteins are essential for viral entry via the angiotensin-converting enzyme 2 (ACE2) receptor. ${ }^{1,2}$ ACE2 receptors are expressed in many cell types, including the neurons and glial cells of the brainstem, raising suspicion of possible neurotropism of SARS-CoV-2. There is substantial evidence of SARS-CoV-2-related neurologic complications through direct and indirect neurotropism.

Below, we review common neurologic complications in patients with coronavirus disease 2019 (COVID-19) resulting from SARS$\mathrm{CoV}-2$ infection.

\section{ACUTE ENCEPHALOPATHY}

\section{Presentation \\ Altered mental status \\ Supportive testing \\ Magnetic resonance imaging (MRI) normal \\ Electroencephalography abnormal (slowing) \\ Cerebrospinal fluid normal, negative for SARS-CoV-2 \\ Treatment \\ Supportive, treat underlying COVID-19}

Encephalopathy is a global cerebral dysfunction associated with infection, fever, drug exposure, and metabolic derangement. This altered functional state is a relatively common presenting symptom of severe COVID-19 disease., ${ }^{3,4}$

Careful examination and appropriate neu- 
rologic workup are necessary in patients with acute encephalopathy that is not explained by their clinical condition. This was highlighted in a series of 13 patients with COVID-19 and unexplained encephalopathy, in whom brain MRI showed leptomeningeal enhancement in $8 \mathrm{pa}$ tients and frontotemporal hypoperfusion in $11 .{ }^{5}$

\section{ACUTE CEREBROVASCULAR DISEASES}

Presentation
Acute presentation with focal motor, sensory,
or speech disturbance
Supportive testing
MRI abnormal, lesion located in a vascular distribution
Treatment
No society guidelines for COVID-19-specific
stroke treatment
Acute ischemic stroke
Consider thrombolytic and endovascular therapy
No society guidelines on stroke prevention
Consider therapeutic anticoagulation on a
case-by-case basis
Acute hemorrhagic stroke (rare)
Standard treatment with blood pressure control
Cerebral venous sinus thrombosis
Standard treatment with full-dose therapeutic
anticoagulation, evaluate for other thrombosis
sites
are expressed in many cell types, including neurons and glial cells of the brainstem

Stroke has been reported in $2.5 \%$ to $6 \%$ of hospitalized patients with COVID-19.4,6,7 In a study of 219 hospitalized patients with COVID-19 in Wuhan, China, those with acute stroke were more likely to be older, present with severe infection, and have cardiovascular risk factors, including a history of stroke. ${ }^{6}$ Intracranial hemorrhage was much less common than acute ischemic strokes.

\section{Acute ischemic stroke}

Cases of acute ischemic strokes were reported during the SARS-CoV and MERS-CoV epidemics. In patients with COVID-19, a case series from New York City reported largevessel ischemic stroke in 5 patients younger than 50 years. $^{7}$ Each presented with acute stroke symptoms with lymphopenia and elevated inflammatory markers on admission laboratory testing, but 2 had no COVID-19 symptoms.

Lupus anticoagulants and prolonged activated partial thromboplastin time have also been frequently reported among hospitalized COVID-19 patients, with a prevalence of $45 \%$ to $91 \%$ for lupus anticoagulants. ${ }^{8,9}$ While there is no clear association between lupus anticoagulants and thrombosis in these studies, a case series reported antiphospholipid antibodies in 3 critically ill COVID-19 patients with bilateral cerebral infarcts in multiple vascular territories. ${ }^{10}$ This suggests that an acquired antiphospholipid syndrome was the underlying cause, but unlike the 5 young patients who had large-artery strokes, ${ }^{?}$ the patients with antiphospholipid antibodies were over 60 years of age.

These reports show that the prevalence of antiphospholipid antibodies varies in patients with COVID-19, but it is likely higher than expected in the general population. As the clinical significance is not yet known, these laboratory values should not be routinely checked in COVID-19 patients without thrombosis.

Other causes of ischemic stroke, such as viral-induced central nervous system vasculitis, have been considered in COVID-19 patients with brain lesions in vascular patterns but without clear cerebrovascular etiology. A postmortem histologic analysis of 3 patients with COVID-19 revealed lymphocytic endotheliitis within the endothelial cells of multiple organs, including the lungs, heart, kidneys, small intestine, and liver. ${ }^{11}$ Endotheliitis can cause microcirculatory vasoconstriction and endothelial dysfunction with consequent ischemia and apoptosis. However, histopathologic analyses of the central nervous system have been limited, and it remains unclear if lymphocytic endotheliitis has been established in COVID-related central nervous system vasculitis.

\section{Venous thromboembolism}

Patients with severe COVID-19 also may be at risk of thromboembolic events from COVID-19-associated coagulopathy. ${ }^{12,13}$ In hospitalized patients with COVID-19, the increased coagulation activity is marked by elevated D-dimer concentrations., ${ }^{3,12,13}$ Further, patients with COVID-19 and cerebrovascular disease had higher D-dimer levels than those without cerebrovascular disease $(6.9 \mathrm{mg} / \mathrm{L}$ vs $0.5 \mathrm{mg} / \mathrm{L}, P<.001) .{ }^{6}$ At this 
time, however, it is unclear if elevated Ddimer levels in patients with COVID-19 are directly associated with either arterial or venous ischemic stroke.

A case series and systematic review ${ }^{14}$ reported 14 cases of cerebral venous sinus thrombosis, with a median of 7 days from initial COVID-19 symptoms to diagnosis of the thrombosis. Initial imaging revealed cerebral venous sinus thrombosis-related intracranial hemorrhage with involvement of the transverse $(75 \%)$, sigmoid $(50 \%)$, and deep venous sinuses $(33 \%)$ at presentation. The mortality rate was high despite therapeutic anticoagulation.

Overall, the incidence of COVID-19-related cerebral venous sinus thrombosis remains much lower than that of acute ischemic stroke. ${ }^{15}$

\section{CENTRAL NERVOUS SYSTEM INFECTIONS}

\section{Encephalitis, meningitis}

Presentation
Headache, nuchal rigidity, seizures, focal neurologic
deficits, plus altered mental status in encephalitis
Supportive testing
MRI abnormal, white matter changes
Electroencephalography normal to abnormal
(slow, with or without focal epileptiform discharges)
Cerebrospinal fluid normal to lymphocytic pleocytosis
with or without elevated protein;
SARs-CoV-2-positive
Treatment
Remains unclear
Role for high-dose corticosteroids?

Encephalitis is characterized by brain inflammation that can cause morbidity and death if left untreated. ${ }^{16}$ In acute viral encephalitis, the virus replicates in brain tissue, leading to significant central nervous system insults. Studies in mice have shown that the human coronavirus can infect neurons and subsequently cause persistent infection in human neural-cell lines. ${ }^{17}$

Ellul et $\mathrm{al}^{18}$ tallied 8 cases of encephalitis from various sources. These patients presented with a range of symptoms, including irritability, confusion, seizures, and nuchal rigidity. Cerebrospinal fluid analysis in 5 patients detected lymphocytic pleocytosis. Most brain

imaging was normal, but electroencephalography completed in 5 patients showed generalized slowing, focal epileptiform discharges, and 1 case of nonconvulsive status epilepticus. No specific treatment was noted in these patients; however, 1 patient responded quickly to high-dose steroids.

The low reported rate of central nervous system infection in patients with COVID-19 is likely an underestimation, as the subtle symptoms of encephalitis may be missed, and performing a lumbar puncture in patients with severe COVID-19 infection requires a substantial risk-benefit consideration.

\section{Postinfectious demyelination}

\section{Presentation \\ Headache, acute neurologic symptoms \\ Supportive testing \\ MRI shows hyperintense fluid-attenuated inversion recovery (FLAIR) lesions with variable enhancement \\ Treatment \\ 2 case reports showed improvement with: \\ 5 days of intravenous immunoglobulin $0.4 \mathrm{~g} / \mathrm{kg} / \mathrm{day}{ }^{19}$ \\ 5 days of intravenous dexamethasone \\ $20 \mathrm{mg} /$ day with a 10-day taper $^{20}$}

Acute disseminated encephalomyelitis is a monophasic, demyelinating disease of the central nervous system characterized by multifocal white matter demyelination in the setting of a rapidly progressive encephalopathy. An antecedent infectious process before the onset of central nervous system symptoms is common; however, the cause is typically only found in a small percentage of cases.

Two cases of probable acute disseminated encephalomyelitis have been reported in the COVID-19 population, with bilateral, extensive, nonenhancing T2-FLAIR signal changes noted in the cerebral white matter, involving the subcortical brain parenchyma and cervical spinal cord. ${ }^{19,20}$ The cerebrospinal fluid was negative for SARS$\mathrm{CoV}-2$ by polymerase chain reaction testing in both cases. One patient was treated with intravenous immunoglobulin and the other with a 5-day course of a high-dose cortico-

\section{The increased coagulation activity is marked by elevated D-dimer concentrations}


steroid with a 10-day taper. Neurologic improvement was noted in both patients.

\section{Acute necrotizing hemorrhagic encephalopathy}

A case report presented details of a SARS$\mathrm{CoV}-2$-infected woman in her late $50 \mathrm{~s}$ who presented with fever, cough, and altered mental status. ${ }^{21}$ Computed tomography (CT) without contrast depicted symmetric hypoattenuation in the bilateral medial thalami with a normal CT angiogram and venogram. MRI demonstrated hemorrhagic, enhancing lesions within the bilateral thalami, medial temporal lobes, and subinsular regions. Cerebrospinal fluid analysis revealed negative bacterial cultures and viral testing. The reason for not testing the cerebrospinal fluid for SARSCoV-2 was not reported. Based on imaging and clinical context, she was given a diagnosis of probable acute necrotizing hemorrhagic encephalopathy and started on intravenous immunoglobulin therapy. No further information is available on her clinical course.

Acute necrotizing hemorrhagic encepha-

The low

reported rate

of central

nervous system

infection

in COVID-19

is likely

an under-

estimation lopathy in children with virulent influenza infections has been well described. ${ }^{22}$ This complication is pathologically distinguished from acute disseminated encephalomyelitis by causing blood-brain barrier breakdown without direct viral invasion or demyelination. ${ }^{23}$

\section{SEIZURES}

Although new-onset seizures in patients who have COVID-19 are rare, 2 cases were reported of acute symptomatic seizures in nonepileptic patients with COVID-19 at Cleveland Clinic. ${ }^{24}$

Two retrospective studies have described electroencephalographic patterns seen in patients with acute COVID-19. ${ }^{25,26}$ One study reported frequent sporadic interictal epileptiform discharges in 22 patients with
COVID-19, using mostly continuous 8-channel electroencephography. ${ }^{25}$

However, this finding was not supported by the other study, which used standard 21-channel recordings. ${ }^{26}$ This study also reported a variety of other electroencephographic findings, including continuous slowing, generalized rhythmic activity, and generalized periodic discharges. ${ }^{26}$

These studies support the high incidence of encephalopathy in hospitalized patients with COVID-19 and the presence of acute symptomatic seizures from an underlying metabolic or toxic process or primary central nervous system insult as a complication of COVID-19.

\section{NEUROMUSCULAR DISORDERS}

\section{Presentation \\ Myalgias \\ Supportive testing \\ Creatine kinase elevated \\ Muscle biopsy shows necrosis \\ Treatment \\ Supportive; remains unclear \\ Role for corticosteroids? \\ Physical therapy}

\section{Critical illness polyneuropathy and myopathy}

Patients in the intensive care unit are at risk of developing severe weakness secondary to critical illness polyneuropathy, critical illness myopathy, or both, with a reported incidence of up to $33 \% .{ }^{27}$ To date, there have been no definitive reports of either disorder in patients with COVID-19. However, a study from Wuhan, China, reported 23 COVID-19 patients with acute muscle injury (defined as myalgia and serum creatine kinase level above 200 $\mathrm{U} / \mathrm{L}){ }^{4}$

Thus, clinicians should suspect critical illness polyneuropathy or critical illness myopathy in patients with COVID-19 presenting with sepsis or complications leading to prolonged mechanical ventilation and intensive care unit length of stay. 


\section{Acute inflammatory demyelinating polyneuropathy}

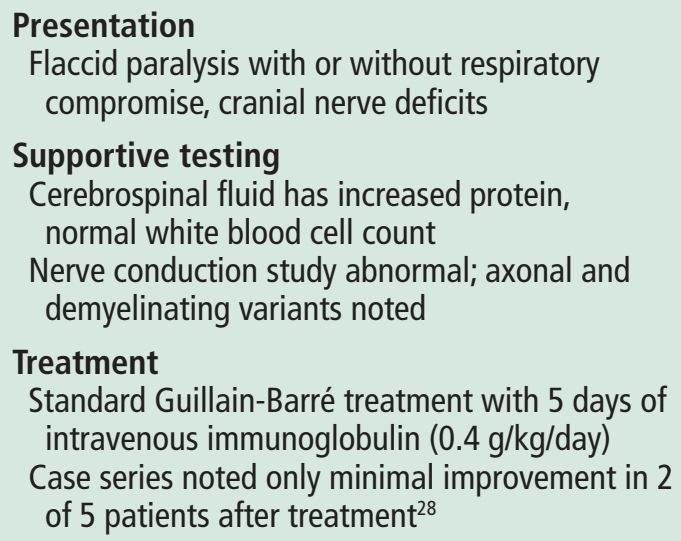

Acute inflammatory demyelinating polyneuropathy (more commonly known as Guillain-Barré syndrome), is an autoimmune demyelinating disorder of the peripheral nervous system usually following an antecedent infection. It is characterized by parathesias, areflexia, and ascending weakness that may lead to respiratory failure.

There are several cases of patients developing acute inflammatory demyelinating polyneuropathy after the onset of COVID-19 symptoms. ${ }^{28-30} \mathrm{~A}$ case series from Italy described 5 patients presenting with paraplegia, facial muscle weakness, and areflexia 5 to 10 days after the onset of COVID-19 symptoms. ${ }^{28}$ Of those, 3 patients had pathognomonic cerebrospinal fluid findings of albuminocytologic dissociation consistent with acute inflammatory demyelinating polyneuropathy. All 5 patients were treated with intravenous immunoglobulins, but after 4 weeks only 1 had been discharged and was able to walk independently.

Although it is important to recognize the classic symptoms of acute inflammatory demyelinating polyneuropathy, a case series from Spain reported 2 rare variants of it, ie, Miller Fisher syndrome and polyneuritis cranialis. ${ }^{30}$

\section{CRANIAL NEUROPATHY}

Olfactory neuropathy

Presentation
Olfactory or taste dysfunction
Supportive testing
Abnormal smell and taste evaluation
Treatment
Supportive; improvement noted by 2 weeks after
symptom onset

Anosmia and dysgeusia are common symptoms associated with COVID-19, and are likely due to the virus directly accessing the olfactory bulb. ${ }^{31}$

A study of 417 patients with mild to moderate COVID-19 symptoms in 12 European hospitals reported sudden-onset olfactory dysfunction in $86 \%$, and gustatory dysfunction in $88 \% .^{32}$ At 2 weeks, $25 \%$ of the patients had recovered both their sense of smell and their sense of taste.

Anosmia and dysgeusia are now recognized as presenting COVID-19 symptoms by the US Centers for Disease Control and Prevention.

\section{NEUROCOGNITIVE IMPAIRMENT}

\section{Presentation \\ Neurocognitive impairments in at least 1 domain after COVID-19 \\ Supportive testing \\ Formal neurocognitive assessment \\ Treatment \\ Consideration for neurorehabilitation programs}

Mental fatigue and mild inattention has been frequently reported in patients with COVID-19. ${ }^{33}$ In 1 series, 179 hospitalized COVID-19 survivors underwent a battery of telephone-administered, standardized neurocognitive, psychiatric morbidity, and quality of life assessments within 2 months of hospital discharge. ${ }^{33}$ Of these, $59 \%$ had neurocognitive impairment in at least 1 function, with moderate impairment of immediate verbal memory and learning in $38 \%$, of verbal fluency in $35 \%$,

\section{At 2 weeks, $25 \%$ had recovered both their sense of smell and of taste}


and of executive function in $6.1 \%{ }^{33}$ Risk factors for neurocognitive impairment included severe COVID-19 infection, hypoxemia requiring mechanical ventilation, hypoperfusion, and increased inflammatory response. Delirium and stress-related symptoms also increased the odds of developing neurocognitive symptoms.
Neurorehabilitation programs have been anecdotally reported to improve neurocognitive symptoms by targeting alertness, sleep problems, and behavior disturbances. ${ }^{34}$ Ongoing monitoring is needed to fully understand the long-term prognosis and psychologic impact of COVID-19.

\section{REFERENCES}

1. Coutard B, Valle C, de Lamballerie X, Canard B, Seidah NG, Decroly E. The spike glycoprotein of the new coronavirus 2019-nCoV contains a furin-like cleavage site absent in CoV of the same clade. Antiviral Res 2020; 176:104742. doi:10.1016/j.antiviral.2020.104742

2. Letko M, Marzi A, Munster V. Functional assessment of cell entry and receptor usage for SARS-CoV-2 and other lineage $B$ beta-coronaviruses. Nat Microbiol 2020; 5(4):562-569. doi:10.1038/s41564-020-0688-y

3. Guan W-J, NI Z-Y, Hu Y, et al. Clinical characteristics of coronavirus disease 2019 in China. N Engl J Med 2020; 382(18):1708-1720. doi:10.1056/NEJMoa2002032

4. Mao L, Jin $\mathbf{H}$, Wang $M$, et al. Neurologic manifestations of hospitalized patients with coronavirus disease 2019 in Wuhan, China. JAMA Neurol 2020; 77(6):1-9. doi:10.1001/jamaneurol.2020.1127

5. Helms J, Kremer S, Merdji H, et al. Neurologic features in severe SARSCoV-2 infection [letter]. N Engl J Med 2020; 382(23):2268-2270. doi:10.1056/NEJMc2008597

6. Li Y, Li M, Wang M, et al. Acute cerebrovascular disease following COVID-19: a single center, retrospective, observational study. Stroke Vasc Neurol 2020; 5(3):279-284. doi:10.1136/svn-2020-000431

7. Oxley TJ, Mocco J, Majidi S, et al. Large vessel stroke as a presenting feature of COVID-19 in the young [letter]. N Engl J Med 2020; 382(20):e60. doi:10.1056/NEJMc2009787

8. Bowles L, Platton S, Yartey N, et al. Lupus anticoagulant and abnormal coagulation tests in patients with Covid-19 [letter]. N Engl J Med 2020; 383(3):288-290. doi:10.1056/NEJMc2013656

9. Harzallah I, Debliquis A, Drénou B. Lupus anticoagulant is frequent in patients with Covid-19 [letter]. J Thromb Haemost 2020; Apr 23. doi:10.1111/jth.14867

10. Zhang Y, Xiao M, Zhang S, et al. Coagulopathy and antiphospholipid antibodies in patients with Covid-19. N Engl J Med 2020; 382(17):e38. doi:10.1056/NEJMc2007575

11. Varga Z, Flammer AJ, Steiger $P$, et al. Endothelial cell infection and endotheliitis in COVID-19. Lancet 2020; 395(10234):1417-1418. doi:10.1016/S0140-6736(20)30937-5

12. Lodigiani C, lapichino G, Carenzo L, et al. Venous and arterial thromboembolic complications in COVID-19 patients admitted to an academic hospital in Milan, Italy. Thromb Res 2020; 191:9-14. doi:10.1016/j.thromres.2020.04.024

13. Klok FA, Kruip MJ, van der Meer NJ, et al. Incidence of thrombotic complications in critically ill ICU patients with COVID-19. Thromb Res 2020; 191:145-147. doi:10.1016/j.thromres.2020.04.013

14. Tu TM, Goh C, Tan YK, et al. Cerebral venous thrombosis in patients with COVID-19 infection: a case series and systematic review. J Stroke Cerebrovasc Dis 2020; 29(12): 105379 . doi:10.1016/j.jstrokecerebrovasdis.2020.105379

15. Asadi-Pooya AA, Simani L. Central nervous system manifestations of COVID-19: a systematic review. J Neurol Sci 2020; 413:116832. doi:10.1016/j.jns.2020.116832

16. Swanson PA 2nd, McGavern DB. Viral diseases of the central nervous system. Curr Opin Virol 2015; 11:44-54. doi:10.1016/j.coviro.2014.12.009

17. Arbour N, Côté G, Lachance C, Tardieu M, Cashman NR, Talbot PJ. Acute and persistent infection of human neural cell lines by human coronavirus OC43. J Virol 1999; 73(4):3338-3350. doi:10.1128/JVI.73.4.3338-3350.1999

18. Ellul MA, Benjamin L, Singh B, et al. Neurological associations of COVID-19. Lancet Neurol 2020; 19:767-783.
doi:10.1016/S1474-4422(20)30221-0

19. Zhang T, Rodricks MB, Hirsh E. COVID-19-associated acute disseminated encephalomyelitis: a case report. medRxiv 2020. https://doi.org/10.1101/202 0.04.16.20068148. Accessed November 17, 2020.

20. Zanin L, Saraceno G, Panciani PP, et al. SARS-CoV-2 can induce brain and spine demyelinating lesions. Acta Neurochir (Wien) 2020; 162(7):14911494. doi:10.1007/s00701-020-04374-x

21. Poyiadji N, Shahin G, Noujam D, et al. COVID-19-associated acute hemorrhagic necrotizing encephalopathy: CT and MRI features. Radiology 2020; 296(2):E119-E120. doi:10.1148/radiol.2020201187

22. Wong AM, Simon EM, Zimmerman RA, Wang HS, Toh CH, Ng SH. Acute necrotizing encephalopathy of childhood: correlation of MR findings and clinical outcome. AJNR Am J Neuroradiol 2006; 27(9):1919-1923. pmid:17032866

23. Rossi A. Imaging of acute disseminated encephalomyelitis. Neuroimaging Clin N Am 2008; 18(1):149-161. doi:10.1016/j.nic.2007.12.007

24. Hepburn M, Mullaguri N, George P, et al. Acute symptomatic seizures in critically ill patients with COVID-19: is there an association? Neurocrit Care 2020; May 28:1-5. doi:10.1007/s12028-020-01006-1

25. Galanopoulou AS, Ferastraoaru V, Correa DJ, et al. EEG findings in acutely ill patients investigated for SARS CoV2/COVID 19: a small case series preliminary report. Epilepsia Open 2020; 5(2):314-324. doi:10.1002/epi4.12399

26. Louis S, Dhawan A, Newey C, et al. Continuous electroencephalopathy (CEEG) characteristics and acute symptomatic seizures in COVID-19 patients. medRxiv 2020. https://doi.org/10.1101/2020.05.26.20114033. Accessed November 17, 2020.

27. de Letter MA, Schmitz PI, Visser LH, et al. Risk factors for the development of polyneuropathy and myopathy in critically ill patients. Crit Care Med 2001; 29(12):2281-2286. doi:10.1097/00003246-200112000-00008

28. Toscano G, Palmerini F, Ravaglia S, et al. Guillain-Barré syndrome associated with SARS-CoV-2. N Engl J Med 2020; 382(26):2574-2576. doi:10.1056/NEJMc2009191

29. Zhao $H$, Shen $D$, Zhou $H$, et al. Guillain-Barré syndrome associated with SARS-CoV-2 infection: causality or coincidence? Lancet Neurol 2020; 19(5):383-384. doi:10.1016/s1474-4422(20)30109-5

30. Gutierrez-Ortiz C, Mendez-Guerrero A, Rodrigo-Rey S, et al. Miller Fisher syndrome and polyneuritis cranialis in COVID-19. Neurology 2020; 95(5):e601-e605. doi:10.1212NVNL.0000000000009619

31. Desforges M, Le Coupanec A, Dubeau P, et al. Human coronaviruses and other respiratory viruses: underestimated opportunistic pathogens of the central nervous system? Viruses 2019; 12(1):14. doi:10.3390/v12010014

32. Lechien JR, Chiesa-Estomba CM, De Siati DR, et al. Olfactory and gustatory dysfunctions as a clinical presentation of mild-to-moderate forms of the coronavirus disease (COVID-19): a multicenter European study. Eur Arch Otorhinolaryngol 2020; 277(8):2251-2261. doi:10.1007/s00405-020-05965-1

33. Méndez R, Balanza-Martinez V, Luperdi SC, et al. Short-term neuropsychiatric outcomes and quality of life in COVID-19 survivors. MedRxiv. doi:10.1101/2020.09.23.20190090

34. Devita M, Bordignon A, Sergi G, et al. The psychological and cognitive impact of Covid-19 on individuals with neurocognitive impairments: research topics and remote intervention proposals. Aging Clin Exp Res 2020. doi:10.1007/s40520-020-01637-6

Address: Sung-Min Cho, DO, Department of Neurology, Neurosurgery, Anesthesiology and Critical Care Medicine, Division of NCCU, Johns Hopkins Medical Institutions, 600 N. Wolfe Street, Phipps 455, Baltimore, MD 21287; csungmi1@jhmi.edu 